\title{
Acute urinary retention secondary to urethral involvement of granulomatosis with polyangiitis
}

\author{
Patrick T. Anderson; ${ }^{1^{*}}$ Stephanie Gottheil, MD, ${ }^{2 *}$ Manal Gabril, MD; ${ }^{3}$ Lillian Barra, MD; ${ }^{2}$ Nicholas Power, $M D^{4}$ \\ 'Department of Surgery, Division of Urology, University of Ottawa, The Ottawa Hospital, Ottawa, ON, Canada; ${ }^{2}$ Department of Medicine, Division of Rheumatology; ${ }^{3}$ Department of Pathology; ${ }^{4}$ Department \\ of Surgery, Division of Urology; Schulich School of Medicine and Dentistry, Western University, London Health Sciences Centre, London, ON, Canada
}

*Co-first authors

Cite as: Can Urol Assoc J 2017;11(1-2):E38-40. http://dx.doi.org/10.5489/cuai.3555 Published online January 12, 2017

\section{Abstract}

Granulomatosis with polyangiitis (GPA, formerly Wegener's granulomatosis) is a systemic necrotizing vasculitis of small- and medium-sized blood vessels, primarily affecting the upper and lower respiratory tracts, as well as the kidneys. Urogenital manifestations of GPA are exceedingly rare and usually respond well to systemic immunosuppressive therapy. Here, we present a case of a 36-yearold female presenting with acute urinary obstruction secondary to urethral GPA involvement in the immediate postpartum period. Special consideration should be given to ruling out malignancy in all patients with a history of GPA and urethral lesions, especially when there is a history of cyclophosphamide treatment.

\section{Introduction}

Granulomatosis with polyangiitis (GPA, formerly Wegener's granulomatosis) is a systemic necrotizing vasculitis of smalland medium-sized blood vessels. ${ }^{1}$ GPA is a rare disease, with an incidence reported between seven and 22 new cases per million per year. ${ }^{2}$ The cause of GPA is not well understood, but GPA is associated with serum anti-neutrophil cytoplasmic antibodies (ANCA), in particular anti-PR3 antibodies, which are commonly used in the diagnostic workup. ${ }^{3}$

GPA primarily affects the upper and lower respiratory tracts and can also cause aggressive glomerulonephritis. ${ }^{4}$ Other common manifestations of GPA include otitis media, sinusitis, peripheral neuropathy, and mucocutaneous lesions, as well as ocular, cardiac, and gastrointestinal involvement. ${ }^{1}$ Genitourinary involvement is rare, being reported in less than $1 \%$ of patients in large-cohort studies. ${ }^{5}$ However, GPA has been described to involve the penis, urethra, prostate, bladder, ureter, and epididymis. Urethral GPA involvement has been reported in eight cases at the time of this review. ${ }^{6,7}$
The most common presenting symptoms of urethral GPA were urinary urgency and frequency. Only one patient presented with obstructive symptoms. ${ }^{8}$ In most cases with urogenital involvement, symptoms were successfully resolved with potent immunosuppressive therapies. ${ }^{6-8}$

Many autoimmune diseases are affected by pregnancy and the puerperium. There have been a number of case reports of GPA presenting initially during pregnancy, with only one case report of a patient relapsing during the initial postpartum period.

\section{Case report}

A 36-year-old female presented to the emergency room in acute urinary retention with difficult urinary catheterization. The patient had a history of GPA, diagnosed six years previously, with sinus and pulmonary involvement requiring induction treatment with methotrexate and prednisone; she had been in remission for five years and had not taken any immunosuppressive medications for four years. At this presentation with urinary retention, she was five months postpartum and had been experiencing pelvic pain, hematuria, sinus congestion with epistaxis, and migratory arthralgias since parturition. As well, she had noticed a gradual decrease in her urinary flow that progressed to being dependent on valsalva maneuver for voiding during the week prior to presentation. To relieve her obstruction, a coude tip urinary catheter was placed with note of obstructing granular urethral growths. The patient was sent home with an indwelling urinary catheter and followup with urology for further investigation of the urethral lesions.

Flexible cystourethroscopy revealed normal-appearing bladder, rigid bladder neck with a few polypoid lesions, and a friable appearance throughout the urethra. The urethra displayed lead pipe characteristics with no coaptation when the urinary catheter was removed (Fig. 1). The urethra was firm to palpation. Biopsies were taken from the bladder neck and urethra. Vaginoscopy was normal 


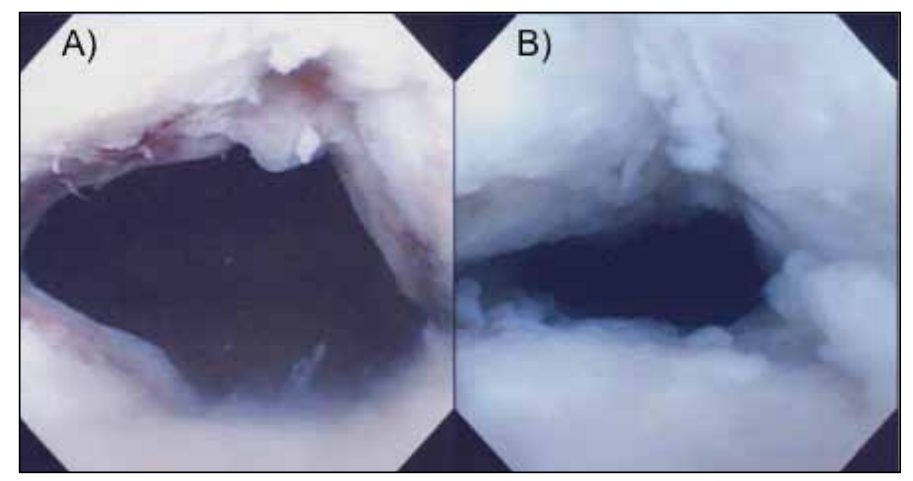

Fig. 1. Urethroscopic appearance (A) prior to treatment with systemic corticosteroid; and (B) following treatment with systemic corticosteroid.

and there was no evidence of inguinal lymphadenopathy on palpation.

\section{Pathology}

Urethral biopsy revealed acute and chronic inflammatory changes with the presence of neutrophils, lymphocytes, and many eosinophils and areas of geographic necrosis (Fig. 2). There was no evidence of malignancy. Bladder neck biopsy also showed severe acute and chronic inflammation with necrotizing epithelioid granulomas. Focal areas of chronic lymphoid cystitis were also noted. There was no evidence of malignancy. Special stains for fungi, bacteria, and acidfast bacilli (PAS-D, Gram, and Ziehl-Neelsen) were negative. These findings supported a diagnosis of relapsed GPA involving the urethra and bladder neck.

\section{Management}

Initial management involved the placement of an indwelling 16-French urinary catheter and referral to rheumatology. The patient was diagnosed with relapsing GPA, given her urethral findings, as well as her sinusitis, arthralgias, and persistently elevated anti-PR3 antibody. She was started on high-dose prednisone. She was also started on methotrexate while awaiting health insurance coverage for rituximab for this severe manifestation of GPA. The Foley catheter was removed six weeks after starting the immunotherapy and the patient is currently being managed with clean intermittent catheterizations.

\section{Discussion}

We report a case of urethral GPA presenting with obstruction and acute urinary retention. Such cases are exceedingly rare, with only eight other cases previously described in the literature. Of these, only one other case presented with obstructive symptoms. ${ }^{8}$ Most cases were successfully managed with immunosuppressive medications, generally cyclophosphamide and prednisone.

It is important to rule out urethral malignancy in any patient presenting with obstructive acute urinary retention and an abnormal appearing urethra. For our patient, this was done through biopsy of the abnormal appearing urethra and bladder neck. Bladder cancer is much more common among patients with a history of GPA than urethral involvement, especially in patients who have undergone cyclophosphamide treatment for their GPA. Our patient did not have this history, but it should be noted that cyclophosphamide exposure is a known risk factor for the development of bladder cancer. ${ }^{9}$ Studies of patients with GPA treated with cyclophosphamide have revealed bladder cancer rates of up to $5 \% .{ }^{10,11}$ Therefore, investigation in these patients should focus on ruling out malignancy prior to pursuing urethral GPA involvement as a cause for obstructive symptoms.

Interestingly, our patient presented with pelvic pain, hematuria, and obstructive urinary symptoms that started immediately postpartum and progressed over the following

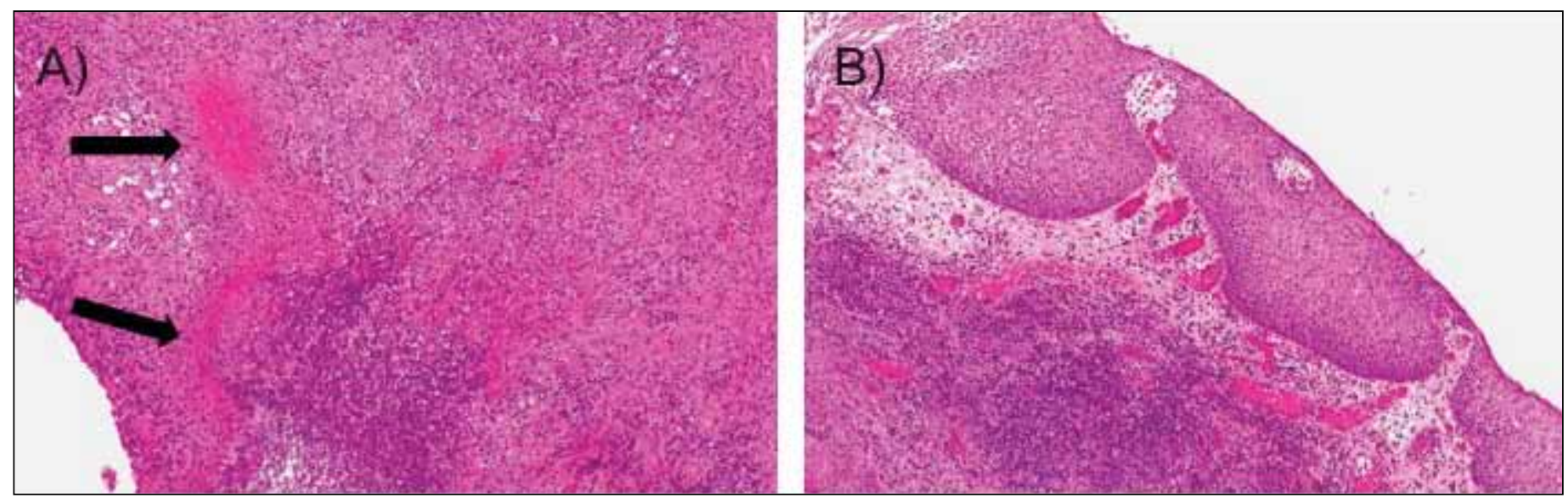

Fig. 2. (A) Sections from urethra and bladder neck show severe acute and chronic inflammation with necrotizing epithelioid granulomas (arrow) with geographical areas of necrosis; $(\boldsymbol{B})$ urothelial hyperplasia shows no evidence of dysplasia or malignancy with underlying acute and chronic inflammation and edema of lamina propria. 
Anderson et al.

months. The association between the postpartum period and GPA relapse has been described in at least one other case. ${ }^{12}$ Further research is ongoing with respect to the role of pregnancy and the puerperium in the reactivation of GPA, as well as the role of maintaining disease surveillance during these periods. ${ }^{13}$ Risk factors for relapse include persistent anti-PR3 positivity, which was present in our patient. ${ }^{12}$

\section{Conclusion}

We have reported a case of a patient presenting with obstructive acute urinary retention secondary to urethral GPA involvement in the early postpartum period. Clinical assessment of patients presenting with obstructive acute urinary retention in the context of a urethral lesion should be aimed at ruling out malignancy. There should be a high index of suspicion for GPA relapse in patients with new symptoms and persistent anti-PR3 positivity. Treatment of urethral GPA involvement should address urinary obstruction and will ultimately involve immunosuppressive therapy.

Competing interests: Dr. Power has participated in clinical trials for Argos Therapeutics. The remaining authors report no competing personal or financial interests.

This paper has been peer-reviewed.

\section{References}

1. Comarmond C, Cacoub P. Granulomatosis with polyangiitis (Wegener): Clinical aspects and treatment. Autoimmun Rev 2014;13:1121-5. https://doi.org/10.1016/i.autrev.2014.08.017

2. Mohammad AJ, Jacobsson LTH, Westman KWA, et al. Incidence and survival rates in Wegener's granulomatosis, microscopic polyangiitis, Churg-Strauss syndrome, and polyarteritis nodosa. Rheumatol Oxf Engl 2009;48:1560-5. https://doi.org/10.1093/rheumatology/kep304

3. Lyons PA, Rayner TF, Trivedi S, et al. Genetically distinct subsets within ANCA-associated vasculitis. N Engl J Med 2012;367:214-23. https://doi.org/10.1056/NEJMoal 108735

4. Schilder AM. Wegener's granulomatosis vasculitis and granuloma. Autoimmun Rev 2010;9:483-7. https://doi.org/10.1016/i.autrev.2010.02.006

5. Huong DL, Papo T, Piette JC, et al. Urogenital manifestations of Wegener granulomatosis. Medicine 1995;74:152-61. htrps://doi.org/10.1097/00005792-199505000-00005

6. Davenport A, Downey SE, Goel S, et al. Wegener's granulomatosis involving the urogenital tract. Br J Urol 1996;78:354-7. https://doi.org/10.1046/i.1464-410X.1996.00166.x

7. Dore $B$, Duriez $P$, Grange $P$, et al. Wegener's granulomatosis with urethral-penile location. A propos of a case. Ann Urol 1990;24:256-8.

8. Zielonka $T M$, Madalinska $M$, Droszzz W, et al. Urethral stricture as unusual complications of Wegener's granulomatosis. Neth J Med 1995;46:236-8. https://doi.org/10.1016/0300-2977(95)81501-5

9. Faurschou M, Mellemkjaer L, Voss A, et al. Prolonged risk of specific malignancies following cyclophosphamide therapy among patients with granulomatosis with polyangiitis. Rheumatology (Oxford) 2015;54:1345-50. https://doi.org/10.1093/rheumatology/keu372

10. Alba MA, Moreno-Palacios J, Beca $\mathrm{S}$, et al. Urologic and male genital manifestations of granulomatosis with polyangiitis. Autoimmun Rev 2015;14:897-902. https://doi.org/10.1016/..autrev.2015.05.012

11. Reinhold-Keller E, Beuge $\mathrm{N}$, Latza $\mathrm{U}$, et al. An interdisciplinary approach to the care of patients with Wegener's granulomatosis: Long- term outcome in 155 patients. Arthritis Rheum 2000;43:1021-32. https://doi.org/10.1002/1529-0131 (200005)43:5<1021::AID-ANR10>3.0.C0;2-J

12. Woydodt A, de Groot K, Bahte $S$, et al. Severe relapse of Wegener's granulomatosis during the early postpartum period. Ann Rheum Dis 2006;63:137. https://doi.org/10.1136/ard.2005.037598

13. Grygiel-Gorniak B, Puszczewicz M. Granulomatosis with polyangiitis in pregnancy — clinical implications and treatment possibilities. Eur Rev Med Pharmacol Sci 2015;13:2331-5.

Correspondence: Dr. Patrick T. Anderson, Department of Surgery, Division of Urology, University of Ottawa, The Ottawa Hospital, Ottawa, ON, Canada; patanderson@toh.ca 